\title{
EL ESTUDIO DE LOS CAMBIOS ENTÉSICOS EN RESTOS HUMANOS DE ARGENTINA. ESTADO ACTUAL Y AVANCES FUTUROS
}

\section{ENTHESEAL CHANGES IN HUMAN SKELETAL REMAINS FROM ARGENTINA. CURRENT KNOWLEDGE AND FUTURE PROSPECTS}

\author{
Rodrigo Zúñiga Thayer ${ }^{*}$ y Jorge Suby² \\ ${ }^{l}$ Facultad de Filosofía y Letras. Universidad de Buenos Aires. Grupo de Investigación en Bioarqueología. Buenos Aires. Argentina. \\ ${ }^{2}$ Instituto de Investigaciones Arqueológicas y Paleontológicas del Cuaternario Pampeano (INCUAPA-CONICET). Universidad \\ Nacional del Centro de la Provincia de Buenos Aires. Grupo de Investigación en Bioarqueología. Quequén. Argentina.
}

\section{PALABRAS CLAVE marcadores de estrés musculoesqueletal; bioarqueología; actividad física}

RESUMEN Durante la última década el análisis de los cambios entésicos se ha incluido como parte de las investigaciones bioarqueológicas de diversas regiones de Argentina. En este trabajo se presenta una síntesis de los principales resultados alcanzados hasta el momento y se proponen algunos caminos para los futuros estudios. Los resultados más recientes desarrollados en Argentina tienen en cuenta, en general, los conocimientos más actuales. Se identifican, sin embargo, tres aspectos a ser considerados: 1) las distintas metodologías de registro empleadas; 2) el reconocimiento y control de los múltiples factores biológicos involucrados en los cambios entésicos, incluyendo la masa corporal, la talla y la robustez diafisial, además del sexo y la edad; y 3) la interpretación multifactorial de sus resultados. Se discuten los alcances que han tenido estos estudios en escalas locales y se proponen nuevos avances considerando interrogantes teóricos y metodológicos. Rev Arg Antrop Biol 21(2), 2019. doi: $10.24215 / 18536387 \mathrm{e} 003$

\section{KEY WORDS musculoskeletal stress markers; bioarchaeology; physical activity}

ABSTRACT Analysis of entheseal changes was included during the last decade as part of bioarchaeological research from different regions of Argentina. This paper presents a synthesis of the main results achieved so far in the study of entheseal changes of archaeological human remains from Argentina. The most recent research takes into consideration the most current knowledge. However, three aspects have to be taken into account: 1) the different recording methodologies; 2) the recognition and control of the multiple biological factors involved in entheseal changes, including body mass, body size, and the diaphyseal robusticity, in addition to sex and age; and 3) the multifactorial interpretation of the results. Local progress is discussed and future prospects are proposed, considering theoretical and methodological issues. Rev Arg Antrop Biol 21(2), 2019. doi: $10.24215 / 18536387 \mathrm{e} 003$
El esqueleto humano está expuesto a presiones fisiológicas y ambientales que pueden producir distintos tipos de modificaciones en su estructura interna y externa. Algunas de estas modificaciones son con frecuencia analizadas en restos humanos a través de los denominados marcadores de estrés ocupacional (markers of occupational stress - MOS) los cuales incluyen a la osteoartrosis, las propiedades geométricas transversales de huesos largos y los cambios entésicos (Dutour, 1992; Jurmain, Alves Cardoso, Henderson y Villotte, 2012; Kennedy, 1989; Ruff, 2008; Ruff, Holt y Trinkaus, 2006). Los MOS han recibido una importante atención en bioarqueología durante los últimos 30 años, en especial debido a las discusiones acerca de su potencial para la reconstrucción e interpreta- ción de patrones de actividad física y modos de vida en poblaciones pasadas (e.g., Dewey, 2018; Jurmain et al., 2012; Lloyd, 2017; Palmer, Quintelier, Inskip y Waters-Rist, 2018; Salega, Henderson y Silva, 2017; Weiss y Jurmain, 2007).

Financiamiento: PICT 2016-0191 otorgado por la Agencia Nacional de Promoción Científica y Tecnológica. Ministerio de Ciencia, Tecnología e Innovación Productiva. Argentina.

*Correspondencia a: Rodrigo Zúñiga Thayer. Grupo de Investigación en Bioarqueología. Unidad de Enseñanza Universitaria Quequén. Universidad Nacional del Centro de la Provincia de Buenos Aires. Calle 508 Nro. 881. CP 7631 Quequén. Buenos Aires. Argentina. E-mail: rzunigat89@gmail.com

Recibido 18 Agosto 2018; aceptado 27 Diciembre 2018

doi: $10.24215 / 18536387 \mathrm{e} 003$ 
Como parte de los MOS, recientemente el análisis de los cambios entésicos fue objeto de particular atención, especialmente a partir de las discusiones acerca de los métodos de registro, su terminología y su posterior interpretación (Henderson y Alves Cardoso, 2013). Paralelamente, se comenzaron a realizar este tipo de análisis en restos humanos de diferentes contextos arqueológicos en Argentina, con diferente grado de avance y distintas metodologías. El objetivo de este trabajo es presentar una síntesis de los principales resultados alcanzados hasta el momento en el estudio de cambios entésicos de restos humanos arqueológicos en Argentina, proponiendo futuras direcciones a la luz de los conocimientos actuales. Inicialmente se detallará el conocimiento de las entesis, su fisiología, su clasificación, la historia reciente de las investigaciones sobre estas estructuras, la terminología empleada y las metodologías disponibles.

\section{Antecedentes generales}

Las entesis son las zonas en los huesos donde se insertan tendones, ligamentos, fascias y cápsulas articulares (Benjamin et al., 2002). Se clasifican en entesis fibrocartilaginosas (EFC) y entesis fibrosas (EF), diferenciadas por las estructuras de interfaz existentes entre el tejido blando y duro (Benjamin y McGonagle, 2001; Benjamin y Ralphs, 1998; Benjamin et al., 2002). Las EFC se encuentran en zonas cercanas a las epífisis (Benjamin y McGonagle, 2001) y poseen cuatro tipos distintos de tejido (Fig. 1, modificada de Villote, 2008): 1) extremidad del tendón o ligamento; 2) zona de fibrocartílago no calcificado; 3) zona de fibrocartílago calcificado; y 4) hueso subcondral (Benjamin et al., 2002). Mientras que la transición entre las dos primeras zonas es gradual, existe un límite claro y regular llamado tidemark entre el fibrocartílago no calcificado y el calcificado, que puede ser observado en el registro bioarqueológico (Benjamin y McGonagle, 2001). La transición entre el fibrocartílago calcificado y el hueso subcondral está compuesta por un sistema complejo de múltiples superposiciones entre ambos tejidos, el cual corresponde al "verdadero" límite entre el tendón o ligamento y el hueso subyacente (Benjamin et al., 2002).

Las EF, por otra parte, se encuentran hacia la mitad de las diáfisis de huesos largos, donde tendones y músculos se insertan directamente en el tejido óseo (Benjamin y McGonagle, 2001). Se clasifican en periostadas y óseas. Las más estudiadas son las óseas, compuestas por fibras colágenas tendinosas que se incorporan a una capa superficial fina de hueso nuevo, el cual es remplazado progresivamente por hueso cortical. A pesar de que no existe un consenso claro sobre la apariencia "normal" de una EF, algunos autores la definen como una superficie cortical lisa (Henderson, 2009; Villotte, 2008).

Las primeras aproximaciones que estudiaron la morfología de las inserciones musculares, y su posible vinculación con la actividad física, fueron realizadas principalmente desde la medicina (Lane, 1888; Schneider, 1956; Testut, 1889) y posteriormente por la arqueología (Angel, 1964, 1966; Hawkes y Wells, 1975; Wells, 1963, 1964, 1965). A partir de las décadas de 1980 y 1990 comenzaron a ser utilizadas con mayor frecuencia en la bioarqueología para proponer interpretaciones acerca de patrones de actividad en poblaciones antiguas (e.g., Dutour, 1986; Hawkey y Merbs, 1995; Kennedy, 1983). Esas modificaciones fueron inicialmente señaladas como activity-induced pathology (Merbs, 1983), evidence for occupation (Kelley y Angel, 1987), skeletal makers of occupational stress (Kennedy, 1989) y "marcador de estrés inducido por actividad" (Hawkey y Street, 1992). Sin embargo, el primer término ampliamente utilizado fue musculoskeletal stress marker (MSM), introducido por Hawkey y Merbs (1995) y definido como una o varias marcas distintivas que ocurren donde un músculo, tendón o ligamento se inserta en el periostio y en el hueso cortical subyacente. Recientemente se adoptó el término de "cambios entésicos" en lugar de MSM, destacando su naturaleza no patológica y debido a la etiología multifactorial de los rasgos morfológicos observables en las inserciones musculares (Jurmain y Villotte, 2010; Santos, Alves Cardoso, Assis y Villotte, 2011; Villotte y Knüsel, 2013).

Estos cambios en la nomenclatura acompañaron las críticas acerca de la especificidad causal de los cambios entésicos, asociados inicialmente a la actividad física, principalmente por el reconocimiento de su etiología multifactorial (Benjamin, Coop y Evans, 1986; Jurmain et al., 


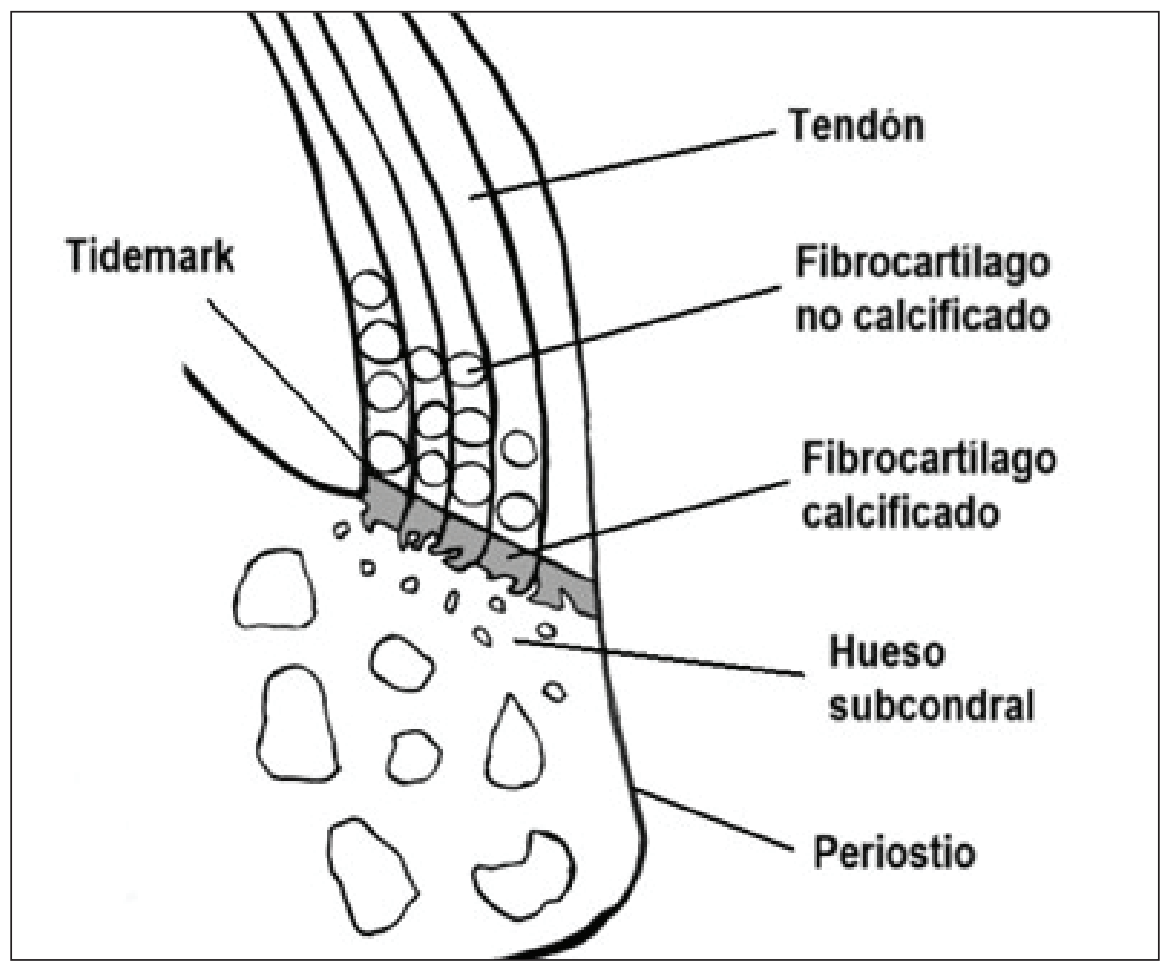

Fig. 1. Zonas tisulares de una entesis FC (Modificado de Villotte, 2008).

2012; Villotte y Knüsel, 2013; Villotte et al., 2016). Actualmente se reconoce que tanto las EF y como las EFC cambian durante el transcurso de la vida, influenciadas por variables biológicas (principalmente la edad, el sexo, la masa corporal y la talla) y según algunos autores posiblemente influenciada por la actividad física, aunque sin alcanzar un consenso claro (Benjamin et al., 1986; Jurmain et al., 2012; Villotte y Knüsel, 2013; Villotte et al., 2016; Weiss,CoronaySchultz,2012).Sobreesteaspecto, Villotte (2008) advirtió que un cambio entésico puede ser causado por distintas actividades físicas que involucren la misma acción muscular. Por lo tanto, en la actualidad se considera que no es posible identificar las actividades específicas que afectan a las entesis (Jurmain et al., 2012; Robb, 1998).

Una línea de investigación relevante para la comprensión del rol de la actividad física en los cambios entésicos es el estudio de conjuntos de restos humanos cuyas ocupaciones se encuentran documentadas (e.g., Alves Cardoso,2008;AlvesCardosoyHenderson, 2010; Cunha y Umbelino, 1995; Milella, 2010; Milella,
Belcastro, Zollikofer y Mariotti, 2012; Niinimäki, 2011; Perréard Lopreno, 2007; Villotte, 2008). Sin embargo, existen discrepancias entre los resultados que posiblemente son causadas por el uso de distintas metodologías de registro y análisis, la significativa influencia de la edad y el uso de distintos criterios para agrupar a los individuos en función de sus profesiones (e.g., combinación de parámetros físicos o categorías socioeconómicas; Cardoso y Henderson, 2013; Henderson, Craps, Caffell, Millard y Gowland, 2013; Perréard Lopreno, Alves Cardoso, Assis, Milella y Speith, 2013).

\section{Propuestas metodológicas de registro}

El primer método de registro sistemático propuesto para el estudio de cambios entésicos, y por lo tanto de gran utilidad para estudios bioarqueológicos, forenses y anatómicos, fue publicado por Hawkey y Merbs (1995). Su sistema distingue tres tipos de remodelaciones óseas (exostosis, robusticidad y lesión de estrés) y utiliza una escala ordinal compuesta por tres 
grados de expresión (intensidad). Sin embargo, el principal problema que presenta este método es su sesgado criterio de registro, ya que no tiene una debida justificación clínica, además de no contar con suficiente respaldo fotográfico y tener un error interobservador elevado (Henderson, 2009; Santos et al., 2011; Villotte, 2008). En 1997, durante el 66th Annual Meeting of Physical Antropologists, se presentó una reevaluación de este método, estableciendo grados de expresión a partir de un conjunto esqueletal identificado del cementerio Pontecagnano (Italia) e introduciendo la dimensión del análisis multivariado (Robb, 1998).

Posteriormente, Mariotti, Facchini y Belcastro (2004, 2007) reevaluaron el método de Hawkey y Merbs (1995) y elaboraron un nuevo sistema de registro a partir de conjuntos esqueletales identificados junto con fotografías y descripciones de los distintos grados de expresión de cada entesis. Mariotti et al. (2004, 2007) sugirieron complementar los resultados con otros tipos de MOS (i.e., osteoartrosis y morfología de la sección transversal de huesos largos). Además, advirtieron que todos los elementos afectados por procesos patológicos deben ser excluidos para evitar falsos positivos. Este método fue luego criticado debido a que la elaboración del sistema de registro no tuvo en consideración antecedentes médicos y también por su elevado error intra- e inter-observador, sobre todo para la robusticidad (ca. 20\%) (Henderson, Mariotti, Pany Kucera, Villotte y Wilczak, 2013; Villotte, 2008).

Teniendo en cuenta estas dificultades, Villotte $(2006,2008,2013)$ propuso por primera vez un método de registro creado a partir información clínica que distingue a las EFC de las EF. A pesar de los avances realizados, este método registra la presencia conjunta de distintos tipos de cambios (i.e., entesofito, porosidad y lesión erosiva) definiendo un grado particular, lo que no permite analizarlos por separado. Las dificultades existentes en estos métodos llevaron a la organización del Workshop on Musculoskeletal Stress Markers (MSM) realizado en Coimbra (Portugal) en 2009 (Santos et al., 2011; http://www.uc.pt/en/cia/events/Occupation_Conference_2016/WorkshopCoimbra), que congregó a 75 investigadores de 21 países. Como uno de sus principales resultados se pro- puso el llamado Método Coimbra (Henderson et al., 2013b, Henderson, Mariotti, Pany Kucera, Villotte y Wilczak, 2015; Henderson, Wilczak y Mariotti, 2016; Santos et al., 2011;), basado en las fortalezas de los criterios propuestos por Mariotti et al. (2004, 2007) y Villotte (2006, 2008, 2013). El Método Coimbra introduce importantes modificaciones en las categorías de cambios entésicos observables: formación ósea, erosión, cambio textural, porosidad fina, macro porosidad y cavitación, que pueden ser clasificados fundamentalmente según el tipo de modificación que produce en: 1) formación de tejido mineralizado, 2) pérdida de la continuidad de la superficie de la entesis, o 3 ) ambas, produciendo un cambio completo de la entesis (Henderson et al., 2015; 2016; Villotte et al., 2016) (Tabla 1).

Este nuevo método además excluye las EF, debido a la escasa información clínica sobre este tipo de entesis y a que no se han encontrado correlaciones entre ellas y la intensidad de la actividad física (Henderson et al., 2013b; Jurmain et al., 2012). Un resumen de los logros alcanzados durante este Workshop fue publicado por Santos et al. (2011). El nuevo Método Coimbra fue presentado a través de una prueba inicial por Henderson et al. (2013b), y formalmente luego por Henderson et al. (2015), al cual se le añadieron correcciones menores (Henderson et al., 2016). Actualmente ya ha sido puesto a prueba y empleado en distintas muestras arqueológicas (e.g., Dewey, 2018; Lloyd, 2017; Palmer et al., 2018; Salega et al., 2017).

Paralelamente, se han desarrollado propuestas metodológicas cuantitativas que comprenden medidas lineares y cálculos de áreas (Churchill y Morris, 1998; Niinimäki, 2011), mediciones 2D mediante capturas de video (Wilczak, 1998), mediciones de perfiles lineares de rugosidad (Henderson y Gallant, 2007) y escaneos 3D de la superficie del área o volumen de las entesis (e.g., Noldner y Edgar, 2013; Nolte y Wilczak, 2013; Pany, Viola y Teschler-Nicola, 2008; Zumwalt, 2006). Aunque el potencial de estos tipos de análisis representa un gran avance para aumentar la precisión en el registro de cambios entésicos y cuantificar su relación con factores que intervienen como la talla y el sexo, los costos de tiempo y dinero continúan siendo los principales obstáculos (Jurmain et al., 2012; Nolte y Wilczak, 2013; Weiss, 2015). 


\section{Antecedentes en Argentina}

Hasta el momento se han realizado en Argentina nueve estudios bioarqueológicos que analizaron los cambios entésicos en restos humanos. Todos ellos estudiaron individuos de poblaciones del pasado, provenientes de la región Central (Salega, 2016; Salega y Fabra, 2017), de Cuyo (Giannotti, 2016; Peralta, 2017), el no- reste argentino -NEA- (Mazza, 2015, 2018), la región Pampeana (Scabuzzo, 2010, 2012) y el sur de Patagonia (Zúñiga Thayer, 2016) (Fig. 2). En la Tabla 2 se describen las características de las muestras analizadas y los principales resultados obtenidos en cada uno de ellos. Con excepción de los trabajos realizados en Córdoba (Salega, 2016; Salega y Fabra, 2017) y en el NEA (Mazza, 2018), los análisis incluyeron

TABLA 1. Sintesis de las categorías y tipos de cambios entésicos, según Henderson et al. $(2015,2016)$ y

Villotte et al. (2016)

\begin{tabular}{|c|c|c|c|c|}
\hline $\begin{array}{c}\text { Categoría de } \\
\text { cambio entésico }\end{array}$ & \multicolumn{2}{|c|}{ Cambios entésicos particulares } & \multirow{2}{*}{$\begin{array}{c}\text { Descripción del cambio } \\
\text { Textura granular, difusa y rugosa al tacto, ocon } \\
\text { estrías alineadas verticalmente. Probablemente } \\
\text { producidas por la mineralización del } \\
\text { fibrocartílago no calcificado. }\end{array}$} & \multirow{2}{*}{\begin{tabular}{|c|}
$\begin{array}{c}\text { Área } \\
\text { afectada }\end{array}$ \\
EFC \\
(zona 2)
\end{tabular}} \\
\hline \multirow{6}{*}{$\begin{array}{l}\text { Formación } \\
\text { de tejido } \\
\text { mineralizado }\end{array}$} & & $\begin{array}{l}\text { Cambio de } \\
\text { textura }\end{array}$ & & \\
\hline & $\begin{array}{c}\text { difusa y } \\
\text { no saliente }\end{array}$ & Erosión & $\begin{array}{l}\text { Depresiones o excavaciones más anchas que } \\
\text { profundas, con márgenes irregulares y piso } \\
\text { discontinuo. Área rugosa al tacto debido a la } \\
\text { formación difusa de tejido. En las EF puede } \\
\text { estar relacionada con la inserción directa de } \\
\text { fibras tendinosas al hueso. }\end{array}$ & $\begin{array}{c}\text { EFC y } \\
\text { EF (zona 1) }\end{array}$ \\
\hline & \multirow{4}{*}{$\begin{array}{c}\text { Protuberancia } \\
\text { ósea aislada }\end{array}$} & Entesofito & $\begin{array}{l}\text { Clara proyección ósea usualmente observada } \\
\text { en el margen de las entesis FC. Tiende a } \\
\text { ser mayor en longitud que en ancho y se } \\
\text { estrecha hacia el vértice. Compuestas por } \\
\text { fibrocartílago calcificado. }\end{array}$ & EFC y EF \\
\hline & & $\begin{array}{l}\text { Margen } \\
\text { saliente }\end{array}$ & $\begin{array}{l}\text { Borde elevado en el margen, no tan } \\
\text { desarrollado como un entesofito. } \\
\text { La longitud es difícil de medir. }\end{array}$ & $\begin{array}{c}\mathrm{EFC} \\
\text { (zona 1) }\end{array}$ \\
\hline & & $\begin{array}{c}\text { Protuberancia } \\
\text { longitudinal }\end{array}$ & $\begin{array}{l}\text { Protuberancia que usualmente afecta toda la } \\
\text { superficie de la entesis, de forma continua } \\
\text { o discontinua. Claramente distinta de la } \\
\text { superficie cortical circundante, pero no es tan } \\
\text { desarrollada y distintiva como un entesofito. } \\
\text { Puede estar acompañada de una superficie } \\
\text { lisa o irregular. }\end{array}$ & EF \\
\hline & & $\begin{array}{c}\text { Protuberancia } \\
\text { amorfa }\end{array}$ & $\begin{array}{l}\text { Protuberancia de tamaño variable, formada } \\
\text { por tejido mineralizado y con forma } \\
\text { aplanada o similar a un montículo. En las } \\
\text { EF la discontinuidad de la superficie cortical } \\
\text { también puede afectar al hueso esponjoso, } \\
\text { mientras que en las FC la discontinuidad } \\
\text { afecta al cartílago calcificado y al hueso } \\
\text { subcondral. }\end{array}$ & EFC y EF \\
\hline
\end{tabular}


TABLA 1. Continuación

\begin{tabular}{|c|c|c|c|c|}
\hline \multirow{6}{*}{$\begin{array}{c}\text { Pérdida de } \\
\text { la continuidad } \\
\text { de la superficie }\end{array}$} & \multirow[b]{2}{*}{ Porosidad } & $\begin{array}{l}\text { Porosidad } \\
\text { fina }\end{array}$ & $\begin{array}{l}\text { Pequeñas perforaciones (diámetro }<1 \mathrm{~mm} \text { ) } \\
\text { redondeadas a ovaladas con bordes suaves } \\
\text { y redondeados, usualmente agrupadas en } \\
\text { áreas localizadas. La apertura es demasiado } \\
\text { pequeña para observar el tejido subcortical. }\end{array}$ & \multirow{2}{*}{$\begin{array}{c}\text { EF y EFC } \\
\text { (zona 2) } \\
\\
\text { EF y EFC } \\
\text { (zona 2) }\end{array}$} \\
\hline & & $\begin{array}{c}\text { Macro } \\
\text { porosidad }\end{array}$ & $\begin{array}{c}\text { Perforaciones (diámetro } \geq 1 \mathrm{~mm} \text { ) redondeadas } \\
\text { a ovaladas con bordes suaves y redondeados. } \\
\text { Se presentan aisladas o en áreas localizadas. } \\
\text { Cuando la abertura es suficientemente grande } \\
\text { es posible observar el tejido subcortical. La } \\
\text { macroporosidad tiene la apariencia de un } \\
\text { foramen vascular. }\end{array}$ & \\
\hline & \multicolumn{2}{|l|}{ Defecto cortical } & $\begin{array}{l}\text { Pérdida ósea focal, generalmente de forma } \\
\text { longitudinal, a menudo remodelada y de } \\
\text { profundidad variable. Usualmente el fondo } \\
\text { de la depresión presenta numerosos poros. } \\
\text { En individuos subadultos, probablemente } \\
\text { relacionados con la actividad desequilibrada } \\
\text { de los osteoclastos y osteoblastos durante la } \\
\text { migración de las entesis. }\end{array}$ & $\mathrm{EF}$ \\
\hline & \multicolumn{2}{|l|}{ Lesión erosiva } & $\begin{array}{l}\text { Destrucción del cartílago mineralizado y del } \\
\text { hueso subcondral, generalmente mayor en } \\
\text { ancho que en profundidad. Sus bordes son } \\
\text { irregulares y pueden presentar remodelación } \\
\text { mínima a significativa. En el fondo de la } \\
\text { depresión se pueden observar trabéculas o } \\
\text { poros. Morfología similar a las erosiones de } \\
\text { las articulaciones sinoviales. }\end{array}$ & $\mathrm{EFC}$ \\
\hline & \multicolumn{2}{|c|}{ Cavitación } & $\begin{array}{l}\text { Lagunas esféricas subcondrales con una } \\
\text { abertura en la superficie más pequeña que el } \\
\text { diámetro de la cavitación y un piso que no } \\
\text { es un canal. Usualmente el tejido esponjoso } \\
\text { está remodelado. }\end{array}$ & $\begin{array}{c}\text { EFC (zona } \\
2 \text { ) }\end{array}$ \\
\hline & \multicolumn{2}{|c|}{ Superficie surcada } & $\begin{array}{c}\text { Surcos rectos, muy delgados, de longitud } \\
\text { variable }(<2-3 \text { mm) en el hueso cortical de } \\
\text { individuos subadultos. Generalmente están } \\
\text { orientados a lo largo del eje longitudinal del } \\
\text { hueso. Probablemente relacionada con el } \\
\text { crecimiento óseo. }\end{array}$ & $\mathrm{EF}$ \\
\hline \multicolumn{3}{|l|}{$\begin{array}{l}\text { Perdida completa } \\
\text { de la morfología } \\
\text { original }\end{array}$} & $\begin{array}{l}\text { Asociado con la formación y/o erosión de } \\
\text { hueso y la porosidad. Se observa con mayor } \\
\text { frecuencia en la tuberosidad mayor del } \\
\text { húmero. }\end{array}$ & EF y EFC \\
\hline
\end{tabular}

EF: entesis fibrosas; EFC: entesis fibrocartilaginosas; Zona 1: contorno de la entesis FC en el que se insertan las fibras de forma más oblicua; Zona 2: resto del contorno y área superficial de la entesis. 


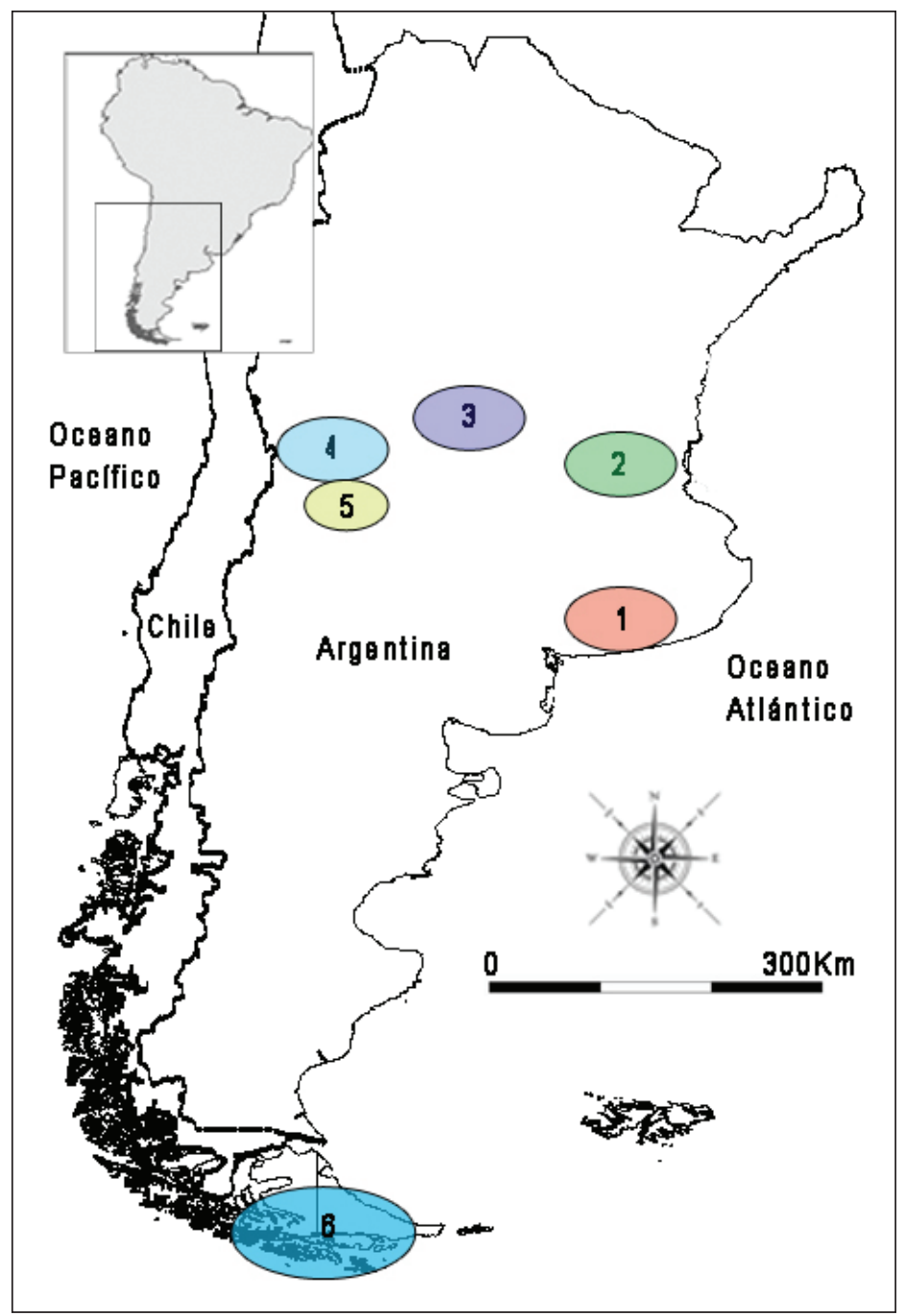

Fig. 2. Localización de las regiones de Argentina de donde provienen los restos humanos en los que se estudiaron cambios entésicos. 1) Scabuzzo, 2010, 2012; 2) Mazza, 2015, 2018; 3) Salega, 2016; Salega y Fabra, 2017; 4) Giannotti, 2016; 5) Peralta, 2017; 6) Zúñiga Thayer, 2016.

pequeñas muestras de esqueletos o conjuntos mezclados de elementos óseos. Si bien algunos estudios a partir de conjuntos mezclados alcanzan un número mínimo de individuos elevado, todos los autores mencionan las dificultades que representa este tipo de conjuntos óseos para obtener información acerca de la lateralidad, la estimación de la edad y el sexo de los individuos.

Siete de los nueve estudios (Tabla 2) incluyeron análisis tanto de miembros superiores como de miembros inferiores. Las únicas excepciones son los estudios de Zúñiga Thayer (2016) y Salega y Fabra (2017), quienes debido a los objetivos planteados analizaron solo las entesis de los miembros superiores e inferiores respectivamente.

La metodología de registro propuesta por Hawkey y Merbs (1995) fue utilizada en cinco de los nueve estudios, en especial porque fueron realizados con anterioridad a la formulación de otros métodos más recientes (Giannotti, 2016; Scabuzzo, 2010, 2012), o como parte de comparaciones con otros métodos (Salega, 2016; Salega y Fabra, 2017). Mazza (2015, 2018) utilizó el método de Mariotti et al. (2007) para registrar la robusticidad y el de Villotte (2006) en su tesis doctoral (Mazza, 2015) para otros tipos de cambios. De igual manera, Zúñiga 
TABLA 2. Estudios de cambios entésicos realizados sobre restos humanos arqueológicos de Argentina, metodologías empleadas y principales resultados alcanzados

\begin{tabular}{|c|c|c|c|c|c|}
\hline Referencia & Cronología & Procedencia & NMI & $\begin{array}{c}\text { Método } \\
\text { empleado }\end{array}$ & Principales resultados \\
\hline $\begin{array}{c}\text { Scabuzzo } \\
(2010)\end{array}$ & $\begin{array}{l}\text { Holoceno } \\
\text { temprano, } \\
\text { medio y } \\
\text { tardío final }\end{array}$ & $\begin{array}{c}\text { Región } \\
\text { pampeana } \\
\text { bonaerense } \\
\text { (Prov. Buenos } \\
\text { Aires) }\end{array}$ & $\begin{array}{l}16 \text { individuos } \\
\text { y } \\
42 \text { elementos }\end{array}$ & $\begin{array}{l}\text { Hawkey } \\
\text { y Merbs } \\
(1995)\end{array}$ & $\begin{array}{c}\text { Diferencias en las demandas } \\
\text { físicas entre los sexos. Mayores } \\
\text { frecuencias de robusticidad en } \\
\text { miembros inferiores de individuos } \\
\text { masculinos, que sugieren } \\
\text { alta actividad deambulatoria. } \\
\text { Continuidad temporal de } \\
\text { actividades. }\end{array}$ \\
\hline
\end{tabular}

\begin{tabular}{ccccc} 
Scabuzzo & Holoceno & Tres Arroyos & Hawkey & \\
temprano y & (Prov. Buenos & 16 individuos & y Merbs \\
medio & Aires) & & División de tareas entre sexos. \\
\hline
\end{tabular}

Individuos con actividades de mayor esfuerzo muscular de los

$\begin{array}{ccccc} & & & & \text { Mariotti et } \\ \text { Mazza } & \text { Holoceno } & \text { del humedal del } & 20 \text { individuos } & \text { al. }(2004, \\ (2015) & \text { tardío } & \text { Paraná Inferior } & \text { y } & 2007) ; \\ & & \text { (Prov. Entre Ríos } & 440 \text { elementos } & \text { Villotte } \\ & & \text { y Buenos Aires) } & \text { óseos } & (2006,2008, \\ & & & 2013)\end{array}$
miembros superiores. División/ especialización del trabajo más allá de cierta diferenciación sexual. Individuos masculinos con mayor estrés biomecánico en los miembros inferiores por actividades deambulatorias que los femeninos.

Ambos individuos cambios en las entesis de los miembros superiores posiblemente

$\begin{array}{ccccc}\text { Giannotti } & \text { S. XVI }- & \begin{array}{c}\text { Mendoza } \\ \text { (Provincia de }\end{array} & \text { 2 individuos } & \begin{array}{c}\text { H Merbs } \\ \text { (2016) }\end{array} \\ \text { XVII } & \text { Mendoza) } & \end{array}$
vinculados al procesamiento de alimentos o traslado de cargas pesadas, y tareas productivas relacionadas con la manufactura textil. Cambios entésicos en los miembros inferiores asociados con actividades de marcha y salto.

Mayor desarrollo de cambios entésicos en miembros superiores e inferiores en individuos de sexo masculino del área de llanuras,

lo que podría asociarse con

$\begin{array}{ccc}\text { Salega } & \text { Holoceno } \\ \text { (2016) } & \text { tardío } & \begin{array}{c}\text { y de llanura } \\ \text { (Provincia de } \\ \text { Córdoba) }\end{array}\end{array}$

Hawkey y Merbs (1995); Henderson et al. (2013, 2015, 2016) actividades de traslado de cargas y desplazamiento a pie, aunque, al considerar los adultos medios, las diferencias sexuales se reducen.

Zúñiga

Thayer

(2016)
Sur de Prov

Holoceno Tierra del Fuego tardío
-Argentina

y Chile
26 individuos $\begin{gathered}\text { Villotte } \\ (2006,200\end{gathered}$

(2006, 2008,

2013)
Los individuos de sexo femenino presentan más cambios entésicos en miembros superiores probablemente causados por la actividad de remo en canoa. 
TABLA 2. Continuación

\begin{tabular}{|c|c|c|c|c|c|}
\hline $\begin{array}{l}\text { Peralta } \\
(2017)\end{array}$ & $\begin{array}{l}\text { Holoceno } \\
\text { tardío }\end{array}$ & $\begin{array}{l}\text { Cuenca media del } \\
\text { valle del Atuel } \\
\text { (Prov. Mendoza) }\end{array}$ & NMI 25 & $\begin{array}{l}\text { Henderson } \\
\text { et al. }(2013 \text {, } \\
2015,2016)\end{array}$ & $\begin{array}{c}\text { Miembros superiores más } \\
\text { afectados que los inferiores. Alta } \\
\text { movilidad para los momentos } \\
\text { posteriores a la introducción de } \\
\text { cultígenos. }\end{array}$ \\
\hline $\begin{array}{l}\text { Salega } \\
\text { y Fabra } \\
(2017)\end{array}$ & $\begin{array}{l}\text { Holoceno } \\
\text { tardío }\end{array}$ & $\begin{array}{c}\text { Región serrana y } \\
\text { de llanura (Prov. } \\
\text { Córdoba) }\end{array}$ & 61 individuos & $\begin{array}{l}\text { Hawkey } \\
\text { y Merbs } \\
(1995) \text { y } \\
\text { Henderson } \\
\text { et al. }(2013 \text {, } \\
\text { 2015, 2016) }\end{array}$ & $\begin{array}{l}\text { Los individuos de llanuras con } \\
\text { mayor desarrollo de cambios } \\
\text { entésicos en miembros inferiores } \\
\text { que los de las sierras. Explicados } \\
\text { tanto desde un punto de vista } \\
\text { adaptativo a la carga biomecánica, } \\
\text { como desde una visión más } \\
\text { clásica referida a la repetición de } \\
\text { actividades físicas exigentes. }\end{array}$ \\
\hline $\begin{array}{l}\text { Mazza } \\
(2018)\end{array}$ & $\begin{array}{l}\text { Holoceno } \\
\text { tardío }\end{array}$ & $\begin{array}{l}\text { Sector meridional } \\
\text { del humedal del } \\
\text { Paraná Inferior } \\
\text { (Prov. Entre Ríos } \\
\text { y Buenos Aires) }\end{array}$ & 73 individuos & $\begin{array}{l}\text { Mariotti et } \\
\text { al. }(2004 \text {, } \\
\text { 2007) }\end{array}$ & $\begin{array}{l}\text { Niveles elevados de cambios } \\
\text { entésicos corresponden a } \\
\text { individuos masculinos, con } \\
\text { mayores longitudes de huesos } \\
\text { largos, índices de robustez } \\
\text { diafisial mayores y con más de } \\
40 \text { años de edad. La actividad } \\
\text { física, el sexo y la edad son los } \\
\text { factores más determinantes en la } \\
\text { morfología de las entesis fibrosas } \\
\text { y fibrocartilaginosas. }\end{array}$ \\
\hline
\end{tabular}

NMI: número mínimo de individuos; Prov.: provincia.

Thayer (2016) utilizó el método propuesto por Villotte (2006, 2008, 2013). Por último, el Método Coimbra (Henderson et al., 2016) fue empleado solo en estudios de restos humanos de las regiones de Centro (Salega, 2016; Salega y Fabra, 2017) y Cuyo (Peralta, 2017).

Todos los estudios tienen en cuenta las diferentes etiologías de los cambios entésicos, con excepción de la masa corporal y la talla, aunque Mazza (2018) emplea la longitud de los huesos largos como proxy del tamaño corporal. Por el contrario, todos los trabajos analizaron sus resultados de acuerdo con la edad de los individuos. También fueron considerados el sexo (Giannotti, 2016; Mazza, 2015; Peralta, 2017; Salega, 2016; Scabuzzo, 2010, 2012; Zúñiga Thayer, 2016), la lateralidad (Giannotti, 2016; Mazza, 2015; Scabuzzo, 2010, 2012; Zúñiga Thayer, 2016), los valores isotópicos (Peralta, 2017; Scabuzzo, 2010), la cronología (Peralta, 2017; Salega, 2016; Scabuzzo, 2010), las ca- racterísticas morfológicas del terreno (Salega y Fabra, 2017) y la robustez diafisial (Mazza, 2018). En algunos casos las frecuencias de cambios entésicos fueron comparadas con frecuencias de lesiones articulares (Giannotti, 2016; Mazza, 2015; Peralta, 2017; Salega, 2016; Scabuzzo, 2010, 2012) o con otras variables métricas de huesos largos (Mazza, 2015, 2018).

Respecto a la edad, la totalidad de los análisis evidenciaron una mayor frecuencia de cambios entésicos en individuos adultos medios y/o mayores que en adultos jóvenes. Esta tendencia general fue interpretada por la mayoría de los autores como consecuencia de un incremento de actividades durante el rango de adultos medios, por factores degenerativos y/o la acumulación de cambios entésicos en el transcurso de la vida de los individuos (Mazza, 2015, 2018; Peralta, 2017; Salega, 2016; Salega y Fabra, 2017; Scabuzzo, 2010, 2012; Zúñiga Thayer, 2016).

En relación con el sexo, todos los estudios, 
excepto el de Mazza (2018) y Zúñiga Thayer (2016), observaron que los individuos masculinos tienen un mayor desarrollo de cambios entésicos que los femeninos, lo que fue atribuido a una actividad física más intensa. Por su parte, Mazza (2018) y Zúñiga Thayer (2016) observaron una tendencia opuesta en algunas entesis de los miembros superiores a partir de conjuntos de pocos individuos provenientes del extremo sur de la bahía de La Plata y de los canales septentrionales del sur de Tierra del Fuego respectivamente.

Ningún estudio realizado observó diferencias estadísticamente significativas en los cambios entésicos según la lateralidad. Los autores sugieren que esta ausencia se debe a la realización de actividades físicas de manera no sistemática (Scabuzzo, 2012), que involucraban movimientos simétricos (Scabuzzo, 2010), a la significativa variación intrapoblacional de patrones de actividad física y/o a la influencia de distintas actividades en los mismos grupos musculares (Mazza, 201; Zúñiga Thayer, 2016).

\section{Discusión y perspectivas futuras}

Una serie de nuevas investigaciones realizadas durante la última década ofrecieron los primeros resultados acerca de los cambios entésicos en sociedades cazadoras-recolectoras y agricultoras del pasado en Argentina. Todos estos trabajos dieron el puntapié inicial para un tipo de estudio sin antecedentes en la región, y por lo tanto de gran mérito. A pesar de estos avances, aun se enfrentan algunos interrogantes teóricos y metodológicos cuyas respuestas podrían resultar útiles, no solo para la interpretación de los procesos biológicos y culturales involucrados, sino para su posterior comparación. Entre estos aspectos, a nuestro criterio se destacan tres: 1) las distintas metodologías de registro empleadas; 2) el reconocimiento y control de los múltiples factores biológicos involucrados en los cambios entésicos, incluyendo la masa corporal, la talla y la robustez diafisial, además del sexo y la edad; y 3) las dificultades en la interpretación de los resultados.

1) Respecto al primer punto, se observa un notorio cambio en las metodologías empleadas, desde los primeros trabajos a los más recientes. Los trabajos iniciales emplearon el método propuesto por Hawkey y Merbs (1995), posteriormente reemplazado por los de Mariotti et al. (2004, 2007) y Villote (2006, 2008, 2013), y más recientemente algunos autores comenzaron a emplear el Método Coimbra. Esta disparidad de métodos, aunque muestra un avance por la incorporación de nuevos criterios, dificulta las comparaciones posibles entre resultados. Probablemente, las muestras analizadas con el método de Hawkey y Merbs (1995) deban ser reanalizados bajo métodos actuales, de manera de corroborar las hipótesis surgidas de estudios anteriores y poder ser compatibles con los obtenidos en otras muestras analizadas con criterios más recientes. Esto se desprende en particular de los resultados logrados por Salega y Fabra (2017), quienes hallaron diferencias importantes entre los resultados obtenidos con el método propuesto por Hawkey y Merbs (1995) y el Método Coimbra (Henderson et al., 2016). La transición a estos nuevos métodos implica abandonar por el momento los análisis sobre las EF, no considerados por el Método Coimbra.

2) Aunque la mayoría de los estudios involucraron el sexo, la edad, la robustez diafisial como variables que podrían influir en los cambios entésicos, la talla y la masa corporal fueron solo ocasionalmente considerados, a pesar de ser relevantes particularmente en los miembros inferiores (Ruff, 2008; Ruff et al., 2006; Weiss, 2004). La incorporación de análisis multifactoriales, incluyendo estas variables morfológicas corporales, podrían producir resultados más ajustados acerca de su posible influencia en el desarrollo de los cambios entésicos estudiados en los restos bioarqueológicos. En contraste, muchos de estos estudios previos coinciden en el análisis de los tipos de prácticas económicas inferidas a partir de la paleodieta y la distribución de las actividades entre hombres y mujeres.

A las diferencias entre trabajos se debe añadir además la influencia que la experiencia del observador tiene sobre este tipo de análisis, lo que podría implicar variaciones intra e interobservador elevadas. Esto es particularmente importante en aquellos casos en los cuales los estudios son realizados por investigadores en formación como parte de sus tesis doctorales, por lo que evaluaciones de este tipo de errores resultan relevantes, incluso en diferentes etapas de una misma investigación. A esto debe añadir- 
se también la falta de uniformidad en las condiciones de observación (e.g., la luz disponible, el uso o no de lupas, etc.), y que pueden incorporar variaciones adicionales a los resultados.

3) Por último, aunque es posible que los cambios entésicos puedan ser influidos por factores bioculturales, la mayor parte de los estudios tienden a ofrecer interpretaciones vinculadas con la actividad física para los resultados encontrados. En este sentido, interpretaciones que incluyan mayor número de factores etiológicos, como los mencionados en el punto 2, permitirán controlar los resultados de los análisis en el futuro. Estudios recientes proponen la implementación de métodos estadísticos multivariados, que como se mencionó más arriba, permiten controlar y aislar varios factores sobre los cambios entésicos (Mazza, 2017; Nikita, 2014, Henderson y Nikita, 2015; Villotte, Churchill, Dutour y Henry-Gambier, 2010).

A estos puntos se añaden avances necesarios que permitan el estudio de cambios entésicos en conjuntos de restos humanos con situaciones particulares, como la interpretación en restos incompletos en los cuales no es posible reconocer todas las entesis de un elemento a lo largo de toda la serie esqueletal, o bien en conjuntos mezclados en los cuales, aunque los elementos pueden estar completos, no es posible asignarlos a un individuo determinado. En este último caso, como consecuencia resulta de mayor dificultad comparar miembros de acuerdo con su lateralidad, interpretar la influencia de la masa corporal, la talla, el sexo o la edad con la misma confiabilidad con que sería posible en individuos completos. Una gran cantidad de sitios arqueológicos en Argentina presentan estas características, por lo que ajustes metodológicos para el tratamiento de este tipo de entierros, como los propuestos por Mazza (2015) y Scabuzzo (2010) serán necesarios en los próximos años.

Otros aspectos parecen haber sido consensuados por la mayoría de los autores que desarrollan este tipo de investigaciones. El término "cambios entésicos" ha sido ampliamente adoptado, al igual que algunos de los conocimientos metodológicos recientes surgidos del Workshop de Coimbra, posiblemente como resultado de las frecuentes vinculaciones entre investigadores de Portugal y Argentina que se dieron durante la última década. Como parte de estas colaboracio- nes, en mayo de 2018 se realizó el primer curso sobre esta temática en Argentina en el marco del IV Taller Nacional de Bioarqueología y Paleopatología (ciudad de Córdoba, 2018), brindado por Charlotte Henderson, una de las más reconocidas especialistas en este tipo de estudios a nivel internacional, y Soledad Salega, una investigadora argentina quien desarrolla trabajos sobre esta temática.

Finalmente, los cambios entésicos de restos humanos de algunas regiones de Argentina aún no han sido explorados. En especial resta avanzar en el estudio de individuos de poblaciones que habitaron el noroeste argentino y el norte de Patagonia. Con la excepción de los restos analizados en la región de Córdoba (i.e., Salega, 2016; Salega y Fabra, 2017), el NEA (i.e., Mazza, 2015, 2018) y Cuyo (i.e., Giannotti, 2016; Peralta, 2016), los trabajos en la Región Pampeana y Patagonia Austral merecen nuevas exploraciones bajo metodologías que reconozcan los conocimientos actuales. Algunos de estos avances ya están en curso, como los que comenzaron a realizarse en Patagonia Austral como parte de la tesis doctoral en curso de uno de nosotros (RZT).

La interacción entre bioarqueólogos dedicados a investigar los cambios entésicos, tanto en Argentina como en Chile (Peralta, Abarca, Giannotti, Herrera Soto y Zúñiga Thayer, 2018) presuponen mayores alcances en los próximos años, cuya interpretación conjunta con otros tipos de análisis, en especial de lesiones y cambios degenerativos articulares, proporcionarán resultados de mayor nivel de confianza acerca de la influencia de la las variables biológicas y culturales en el desarrollo de los cambios entésicos en restos humanos.

\section{AGRADECIMIENTOS}

A los editores de la Revista Argentina de Antropología Biológica y a dos revisores anónimos, cuyos comentarios colaboraron significativamente a mejorar el contenido de este trabajo.

\section{LITERATURA CITADA}

Alves Cardoso, F. (2008). A portrait of gender in two 19th and 20th Portuguese populations: a paleopathology perspective (Tesis doctoral). Department of Archaeology, Durham University, Durham. 
Alves Cardoso, F. y Henderson, C. Y. (2010). Enthesopathy formation in the humerus: data from known age-atdeath and known occupation skeletal collections. American Journal of Physical Anthropology, 141, 550-560. doi:10.1002/ajpa.21171

Angel, J. L. (1964). The reaction area of the femoral neck. Clinical Orthopaedics and Related Research, 32, 130142. doi: 10.1097/00003086-196400320-00022

Angel, J. L. (1966). Early skeletons from Tranquility, California. Smithsonian Contributions to Anthropolology, 2, 1-19. doi:10.5479/si.00810223.2.1

Benjamin, M., Copp, L. y Evans, E. J. (1986). The histology of tendon attachments to bone in man. Journal of Anatomy, 149, 89-100.

Benjamin, M. y McGonagle, D. (2001). The anatomical basis for disease localisation in seronegative spondyloarthropathy at entheses and related sites. Journal of Anatomy, 199, 503-526. doi:10.1046/j.14697580.2001.19950503.x

Benjamin, M. y Ralphs, J. R. (1998). Fibrocartilage in tendons and ligaments. An adaptation to compressive load. Journal of Anatomy, 193, 481-494. doi:10.1046/j.14697580.1998.19340481.x

Benjamin, M., Kumai, T., Milz, S., Boszczyk, B. M., Boszczyk, A. A. y Ralphs, J. R. (2002). The skeletal attachment of tendons-tendon "entheses". Comparative biochemistry and physiology. Part A: molecular \& integrative physiology, 133, 931-945. doi:10.1016/s10956433(02)00138-1

Cardoso, F. A., y Henderson, C. (2013). The categorisation of occupation in identified skeletal collections: a source of bias? International Journal of Osteoarchaeology, 23 (2), 186-196. doi:10.1002/oa.2285

Churchill, S. E. y Morris, A. G. (1998). Muscle marking morphology and labour intensity in prehistoric Khoisan foragers. International Journal of Osteoarchaeology, 8(5), 390-411. doi:10.1002/(sici)10991212(1998090)8:5<390::aid-oa435>3.0.co;2-n

Cunha, E. y Umbelino, C. (1995). What can bones tell about labour and occupation: the analysis of skeletal markers of occupational stress in the Identified Skeletal Collection of the Anthropological Museum of the University of Coimbra (preliminary results). Antropologia Portuguesa, 13, 49-68.

Dewey, J. K. (2018). Evaluating entheseal changes from a commingled and fragmentary population: Republic Groves (Tesis doctoral). Florida Atlantic University, Florida.

Dutour, O. (1986). Entheses (lesions of muscular insertions) as indicators of the activities of Neolithic Saharan populations. American Journal of Physical Anthropology, 71, 221-224. doi:10.1002/ajpa.1330710209

Dutour, O. (1992). Activités physiques et squelette humain: le difficile passage de l'actuel au fossile. Bulletins et Mémoires de la Société d'Anthropologie de Paris n.s., 4 (3), 233-241. doi:10.3406/bmsap.1992.2319

Giannotti, P. S. (2016). Marcadores de estrés ocupacional en poblaciones históricas del norte de Mendoza (s. XVIXVII): Primeros resultados exploratorios. Comechingonia: Revista de Arqueología, 20 (1), 81-110.

Hawkes S. C. y Wells, C. (1975). Crime and punishment in an anglo-saxon cemetery? Antiquity, 49, 118-122. doi:10.1017/s0003598x00063481

Hawkey, D. E. y Street, S. (1992). Activity-induced stress markers in prehistoric human remains from the eastern Aleutian Islands. American Journal of Physical Anthropology [Suppl], 14, 89.

Hawkey, D. E. y Merbs, C. F. (1995). Activity-induced musculoskeletal stress markers (MSM) and subsistence strategy changes among ancient Hudson Bay Eskimos. International Journal of Osteoarchaeology, 5, 324-338. doi:10.1002/oa.1390050403

Henderson, C. Y. (2009). Musculo-skeletal stress markers in bioarchaeology: indicators of activity levels or human variation? A re-analysis and interpretation (Tesis doctoral). Durham University, Durham.

Henderson, C. Y. y Alves Cardoso, F. (2013). Special issue entheseal changes and occupation: technical and theoretical advances and their applications. International Journal of Osteoarchaeology, 23(2), 127-251. doi:10.1002/oa.2298

Henderson, C. Y. y Gallant A. J. (2007). Quantitative recording of entheses. Paleopathology Newsletter 137, 7-12.

Henderson, C. y E. Nikita. 2015. Accounting for multiple effects and the problem of small sample sizes in osteology: A case study focussing on entheseal changes. Archaeological Antropological Science, 8 (4), 805-817. doi:10.1007/s12520-015-0256-1

Henderson, C. Y., Craps, D. D., Caffell, A. C., Millard, A. R. y Gowland, R. (2013a). Occupational mobility in 19th century rural England: the interpretation of entheseal changes. International Journal of Osteoarchaeology, 23 (2), 197-210. doi:10.1002/oa.2286

Henderson, C. Y., Mariotti, V., Pany Kucera, D., Villotte, S. y Wilczak, C. (2013b). Recording specific features of fibrocartilaginous entheses: preliminary results of the Coimbra standard method. International Journal of $\mathrm{Os}_{\mathrm{s}}$ teoarchaeology, 23, 152-162. doi:10.1002/oa.2287

Henderson, C. Y., Mariotti, V., Pany Kucera, D., Villotte, S. y Wilczak, C. (2015). The new 'Coimbra Method': A biologically appropriate method for recording specific features of fibrocartilaginous entheseal changes. International Journal of Osteoarchaeology, 26 (5), 925-932. doi:10.1002/oa.2477

Henderson, C. Y., Wilczak, C. y Mariotti, V. (2016). Commentary: An update to the new Coimbra method for recording entheseal changes. International Journal of Osteoarchaeology, 27 (3), 521-522. doi:10.1002/oa.2548

Jurmain, R. y Villotte, S. (2010). Terminology. Entheses in medical literature: a brief review. En Workshop in Musculoskeletal Stress Markers (MSM): Limitations and Achievements in the Reconstruction of Past Activity Patterns. Recuperado de http://www.uc.pt/en/cia/msm/ msm_after.

Jurmain, $\bar{R}$., Alves Cardoso, F., Henderson, C. Y. y Villotte, S. (2012). Bioarchaeology's holy grail: the reconstruction of activity. En A. L. Grauer (Ed.). A Companion to Paleopathology. Chichester, Reino Unido: WileyBlackell. doi:10.1002/9781444345940.ch29

Kelley, J. O. y Angel, J. L. (1987). Life stresses of slavery. American Journal of Physical Anthropology, 74, 199211. doi:10.1002/ajpa.1330740208

Kennedy, K. A. R. (1983). Morphological variations in ulnar supinator crests and fossae as identifying markers of occupational stress. Journal of Forensic Sciences, 28, 871-876. doi:10.1520/jfs $11594 \mathrm{j}$

Kennedy, K. A. R. (1989). Skeletal markers of occupational stress. En M. Y. Iscan, y K. A. R. Kennedy (Eds.). Reconstruction of life from the skeleton (pp. 129-160). New York, Estados Unidos:Wiley-Liss.

Lane, W. A. (1888). The anatomy and physiology of the shoemaker. Journal of Anatomy and Physiology, 22, 593-628.

Lloyd, D. (2017). Activity patterns and division of labor at a southeastern Tennessee late mississippian site: Toqua. (Tesis doctoral). Illinois State University, Illinois. 
Mariotti, V., Facchini, F. y Belcastro, M. G. (2004). Enthesopathies. Proposal of a standardized scoring method and applications. Collegium Antropologicum, 28, 145-159.

Mariotti, V., Facchini, F. y Belcastro, M. G. (2007). The study of Entheses: proposal of a standardised scoring method for twenty-three entheses of the postcranial skeleton. Collegium Antropologicum, 31, 191-313.

Mazza, B. (2015). Diferenciaciones sociales en el humedal del Paraná inferior: un abordaje desde los comportamientos mortuorios y la biomecánica en sociedades cazadoras-recolectoras de fines del Holoceno tardio (Tesis doctoral). Facultad de Filosofía y Letras, Universidad de Buenos Aires, Buenos Aires.

Mazza, B. (2017). Una aproximación estadística al estudio de los cambios entésicos empleados para evaluar diferencias sexuales en restos humanos. Revista del Museo de Antropología, 10 (2), 145-156. doi:10.31048/1852.4826.v10.n2.16836

Mazza, B. (2018). Entheseal changes among late Holocene hunter-gatherers from the southern extreme of La Plata basin (Argentina). Archaeological and Anthropological Sciences, 10, 1-21. doi:10.1007/s12520-018-0638-2

Merbs, C. F. (1983). Patterns of activity-induced pathology in a canadian inuit population. Archaeological Survey of Canada, 119, 120-128. doi:10.2307/j.ctv173qw

Milella, M. (2010). Skeletal markers of activity: methodological and interpretative reflections after the study of the whole Frassetto Sassari identified skeletal collections (Tesis doctoral). University of Bologna, Bologna.

Milella, M., Belcastro, M. G., Zollikofer, C. P. y Mariotti, V. (2012). The effect of age, sex, and physical activity on entheseal morphology in a contemporary Italian skeletal collection. American Journal of Physical Anthropology, 148, 379-388. doi:10.1002/ajpa.22060

Nikita, E. (2014). The use of generalized linear models and generalized estimating equations in bioarchaeological studies. American Journal of Physical Anthropology, 153, 473-483. doi:10.1002/ajpa.22448

Niinimäki, S. (2011). What do muscle marker ruggedness scores actually tell us? International Journal of $\mathrm{OS}_{\mathrm{S}}$ teoarchaeology, 21, 292-299. doi:10.1002/oa.1134

Noldner, L. K. y Edgar, H. J. (2013). 3D representation and analysis of enthesis morphology. American Journal of Physical Anthropology, 152, 417-24. doi:10.1002/ ajpa.22367

Nolte, M. y Wilczak, C. (2013). Three-dimensional surface area of the distal biceps enthesis, relationship to body size, sex, age and secular changes in a 20th Century American sample. International Journal of Osteoarchaeology, 23, 163-74. doi:10.1002/oa.2292

Palmer, J. L. A., Quintelier, K., Inskip, S. y Waters-Rist, A. L. (2018). A comparison of two methods for recording entheseal change on a post-medieval urban skeletal collection from Aalst (Belgium). Archaeometry. En prensa.

Pany, D., Viola, T. B. y Teschler-Nicola M. (2008). Analysis of musculoskeletal stress markers and joint disease on the early medieval skeletons from Thunau. American Journal of Physical Anthropology, 135, 167-168. doi:10.1002/ajpa.20806

Peralta, E. A. (2017). Cambios y continuidades en la movilidad humana a finales del holoceno tardío: cambios entésicos, lesiones articulares e isótopos estables en el sur de Mendoza. Revista del Museo de Antropología, 10 (2), 157-166. doi:10.31048/1852.4826.v10.n2.16797

Peralta, E. A., Abarca, V., Giannotti P. S., Herrera Soto M. J. y Zúñiga Thayer, R. (2018). Cambios entésicos y patrones de actividad: Potencialidades y Limitaciones en estudios bioarqueológicos. En M. Fabra, P. Novellino,
M. Arrieta, M. S. Salega, C. V. González y A. Tavarone (Comps.). Libro de Resúmenes del IV Taller Nacional de Bioarqueología y Paleopatología. Córdoba, Argentina: Universidad Nacional de Córdoba.

Perréard Lopreno G. (2007). Adaptation structurelle des os du membre supérieur et de la clavicule à l'activité: analyse de l'asymétrie des propriétés géométriques de sections transverses et de mesures linéaires dans une population identifiée (collection SIMON) (Tesis doctoral). Université de Genève, Genève.

Perréard Lopreno, G., Alves Cardoso, F., Assis, S., Milella, M. y Speith, N. (2013). Categorization of occupation in documented skeletal collections: Its relevance for the interpretation of activity-related osseous changes. International Journal of Osteoarchaeology, 23 (2), 175185. doi:10.1002/oa.2301

Robb, J. (1998). The interpretation of skeletal muscle sites: a statistical Approach. International Journal of $\mathrm{Os}$ teoarchaeology, 8, 363-377. doi:10.1002/(sici)10991212(1998090)8:5<363::aid-oa438>3.0.co;2-k

Ruff, C., Holt, B. y Trinkaus, E. (2006). Who's afraid of the big bad Wolff?: "Wolff's law" and bone functional adaptation. American Journal of Physical Anthropology, 129 (4), 484-498. doi:10.1002/ajpa.20371

Ruff, C. B. (2008). Biomechanical analyses of archaeological human skeletons. En M. Katzenberg y S. Saunders (Eds.). Biological anthropology of the human skeleton. (pp. 183-206). New York, Estados Unidos: John Wiley \& Sons. doi:10.1002/9780470245842.ch6

Salega, S. (2016). Prácticas cotidianas, niveles de actividad fisica y modos de vida en poblaciones del sector austral de las Sierras Pampeanas durante el Holoceno Tardio (Tesis doctoral). Facultad de Filosofía y Humanidades, Universidad de Córdoba, CórSalega, S. y Fabra, M. (2017). Variaciones del terreno y cambios entesiales en poblaciones prehispánicas de Córdoba (Argentina). Revista del Museo de Antropología, 10 (2), 131-136. doi:10.31048/1852.4826.v10.n2.16820

Salega, S., Henderson, C. y Silva, A. M. (2017). Cambios entesiales en Portugal: un estudio diacrónico utilizando el nuevo Método Coimbra. Revista del Museo de Antropología, 10 (2), 137-144. doi:10.31048/1852.4826. v10.n2.1682

Santos, A. L., Alves Cardoso, F., Assis, S. y Villotte, S. (2011). The Coimbra workshop in musculoskeletal stress markers (MSM): An annotated review. Antropologia Portuguesa, 28, 135-161. doi:10.14195/21827982 28_5

Scabuzzo, C. (2010). Actividad, patología y nutrición de los cazadores-recolectores pampeanos (Tesis doctoral). Facultad de Ciencias Naturales y Museo, Universidad Nacional de La Plata, La Plata.

Scabuzzo, C. (2012). Estudios bioarqueológicos de marcadores de estrés ocupacional en cazadores recolectores pampeanos del Holoceno Temprano-medio. Análisis de la serie esqueletal de Arroyo Seco 2. Revista Argentina de Antropología Biológica, 14 (1), 17-31.

Schneider, H. (1956). Zur struktur der sehnenansatzzonen. Zeitschrift für anatomie und entwicklungsgeschichte, 119, 431-456. doi:10.1007/bf00522699

Testut, L. (1889). Recherches anthropologiques sur le squelette quaternaire de Chancelade (Dordogne). Lyon, France: Pitrat aîné. doi:10.3406/linly.1889.16318

Villotte, S. (2006). Connaissances Médicales actuelles, cotation des enthésopathies: nouvelle méthode. Bulletins et Mémoires de la Société d'Anthropologie de Paris n.s., $18,65-85$.

Villotte, S. (2008). Enthésopathies et activités des hommes 
préhistoriques-Recherche méthodologique et application aux fossiles européens du Paléolithique supérieur et du Mésolithique (Tesis doctoral). Université Sciences et Technologies-Bordeaux I, Bordeaux.

Villotte, S. (2013). Practical protocol for scoring the appearance of some fibrocartilaginous entheses on the human skeleton. Recuperado de http://www.academia. edu/1427191/Practical protocol for scoring the appearance_of_some_fibrocartilaginous_entheses_on the human skeleton.

Villotte, S., Churchill, S. E., Dutour, O. y Henry-Gambier, D. (2010). Subsistence activities and the sexual division of labor in the european upper paleolithic and mesolithic: evidence from upper limb enthesopathies. Journal of Human Evolution, 59, 35-43. doi: 10.1016/j. jhevol.2010.02.001

Villotte, S. y Knüsel, C. (2013). Understanding entheseal changes: definition and life course changes. International Journal of Osteoarchaeology, 23, 135-146. doi:10.1002/oa.2289

Villotte, S., Assis, S., Alves Cardoso, F., Henderson, C. Y., Mariotti, V., Milella, M., Pany Kucera, D., Speith, N., Wilczak, C. y Jurmain, R. (2016). In search of consensus: terminology for entheseal changes (EC). International Journal of Paleopathology, 13, 49-55. doi:10.1016/j.ijpp.2016.01.003

Weiss, E. (2004). Understanding muscle markers: lower limbs. American Journal of Physical Anthropology, 125, 232-238. doi:10.1002/ajpa.10397

Weiss, E. (2015). Examining activity patterns and biological confounding factors: differences between fibrocartilaginous and fibrous musculoskeletal stress markers. In- ternational Journal of Osteoarchaeology, 25, 281-288. doi:10.1002/oa.2290

Weiss, E., Corona, L. y Schultz, B. (2012). Sex differences in musculoskeletal stress markers: problems with activity pattern reconstructions. International Journal of Osteoarchaeology, 22 (1), 70-80. doi:10.1002/oa.1183

Weiss, E. y Jurmain, R. (2007). Osteoarthritis revisited: a contemporary review of aetiology. International Journal of Osteoarchaeology, 17, 437-450. doi:10.1002/ oa. 889

Wells, C. (1963). Hip disease in ancient man. Report of three cases. Journal of Bone and Joint Surgery, 45, 790-791. doi:10.1302/0301-620x.45b4.790

Wells, C. (1964). Bones, bodies, and disease. Evidence of disease and abnormality in early man. New York, Estados Unidos: Praeger.

Wells, C. (1965). Disease of the knee in anglo-saxons. Medical and Biological Illustration, 15, 100-107.

Wilczak, C. A. (1998). Consideration of sexual dimorphism, age, and asymmetry in quantitative measurements of muscle insertion sites. International Journal of Osteoarchaeology, 8, 311-325. doi:10.1002/(sici)10991212(1998090)8:5<311::aid-oa443>3.0.co;2-e

Zumwalt, A. C. (2006). The effect of endurance exercise on the morphology of muscle attachment sites. Journal of Experimental Biology, 209, 444-454. doi:10.1242/ jeb.02028

Zúñiga Thayer, R. (2016). Aproximación a la actividad de remo en canoa: Un estudio de cambios entésicos en miembro superior de individuos del antiguo territorio yagan (Tesis de pregrado). Facultad de Ciencias Sociales, Universidad de Concepción, Concepción. 